Габдулов Ерканат Кайратулы, Магистрант

Восточно-Казахстанский Государственный

Технический Университет имени Д.Серикбаева

г. Усть-Каменогорск, Казахстан. E-mail: erkanat-1995@mail.ru

ORCID ID 0000-0001-5495-4246

\title{
РАЗРАБОТАТЬ МЕТОДИКУ РАСЧЕТОВ БЕТОННЫХ КОНСТРУКЦИЙ С ИСПОЛЬЗОВАНИЕМ ОБЪЁМНЫХ КОНЕЧНЫХ ЭЛЕМЕНТОВ
}

\author{
Yerkanat Kairatuly Gabdulov \\ D.Serikbayev East Kazakhstan State Technical University \\ City Ust'-Kamenogorsk, the Republic of Kazakhstan \\ E-mail: erkanat-1995@mail.ru \\ ORCID ID 0000-0001-5495-4246

\section{DEVELOP A METHODOLOGY FOR CALCULATING THE STRENGTH OF CONCRETE USING VOLUME FINITE EMEMENTS}

\begin{abstract}
Annotation: This article describes the achievements in the numerical method of solving problems of structural mechanics and resistance of materials. The analysis of modern computer Software Systems was carried out. The analysis of the capabilities of modern PC Comsol Multiphysics and mathematical models implemented in it to solve the problems of calculation of building structures was described.
\end{abstract}

Keywords: Finite element method, FEM, numerical method, strength criterion model.

Аннотация: Данная статья описывает достижения в численном методе решений задач строительной механики и сопротивления материалов. Был проведен анализ современных вычислительных Програмных Комплексов. Описывает анализ возможностей современного ПК Comsol Multipysycs и математические модели реализованные в нем для решения задач расчёта строительных конструкиий.

Ключевые слова: Метод конечных элементов, МКЭ, численный метод, модель критерия прочности.

В настоящее время реализуются высоко технологические проекты со сложной геометрией и работой конструкции, поэтому предъявленные жесткие требования по прочности и надёжности. Это предпологает выполнение разнообразных сложных и точных расходов, основанных на подробных математических моделях которые требуют применения численных методов. В настоящее время наибольшее распространение получил численный метод, реализованный на основе метода конечных элементов (МКЭ). Этот метод реализован в таких программных комплексах как COMSOL Multiphysics, ABAQUS и ANSYS.

Рассмотрим возможности ПК COMSOL Multiphysics который позволяет реализовать сложные математические модели, в том числе и для расчета строительных конструкций. ПК COMSOL имеет главный интерфеис, а также ряд модулей расширяющий его возможности. В работе рассматриваются математические модели поведения и разрушения бетона при многоосном нагружении.

В этом пакете реализованы 3 математические модели критериев прочности, описывающие разрушение бетона под нагрузкой.

1.Модель критерия прочности Вильяма-Варнке.

2.Модель критерия прочности Бреслер-Пистер. 
3.Модель критерия прочности Нильс Сааби Оттосена

\section{Bresler-Pister Criterion}

Критерий Бреслера-Пистера изначально был разработан для того чтобы прогнозировать разрушение бетона при многоосных напряжениях. Этот критерий отказа является расширением Критерия Друкера-Прагера для хрупких материалов и может быть выражен как:

$$
\begin{gathered}
\mathrm{F}_{\mathrm{y}}=\sqrt{\mathrm{J}_{2}+\kappa_{1}} \mathrm{I}_{1}^{2}+\kappa_{2} \mathrm{I}_{1}+\kappa_{3}=0 \\
\kappa_{1}, \kappa_{2}, \kappa_{3} \text { - параметры материала }
\end{gathered}
$$

Этот критерий также может быть записан, перспективе одноосной прочности на сжатие fс октаэдрические нормальные и касательные напряжения

$$
\begin{aligned}
& \sigma_{\text {oct } /}=\mathrm{I}_{1} / 3 \mathrm{and} \tau_{\text {oct }}=\sqrt{2 \mathrm{~J}_{2} / 3} \\
& \text { Получаем уравнение } \\
& \mathrm{F}_{\mathrm{y}}=\frac{\tau_{\text {oct }}}{\mathrm{f}_{\mathrm{c}}}-\mathrm{a}+\mathrm{b} \frac{\sigma_{\text {oct }}}{\mathrm{f}_{\mathrm{c}}}-\mathrm{c} \frac{\sigma_{\text {oct }}^{2}}{\mathrm{f}_{\mathrm{c}}^{2}}=0
\end{aligned}
$$

Здесь, параметры a, b, и с получены от одноосного обжатия, одноосного испытания на растяжение и двухосное сжатие, соответственно. Октаэдрические нормальные напряжения $\sigma_{\text {осt }}$ считается положительным при растяжении, a fc принимается положительным.

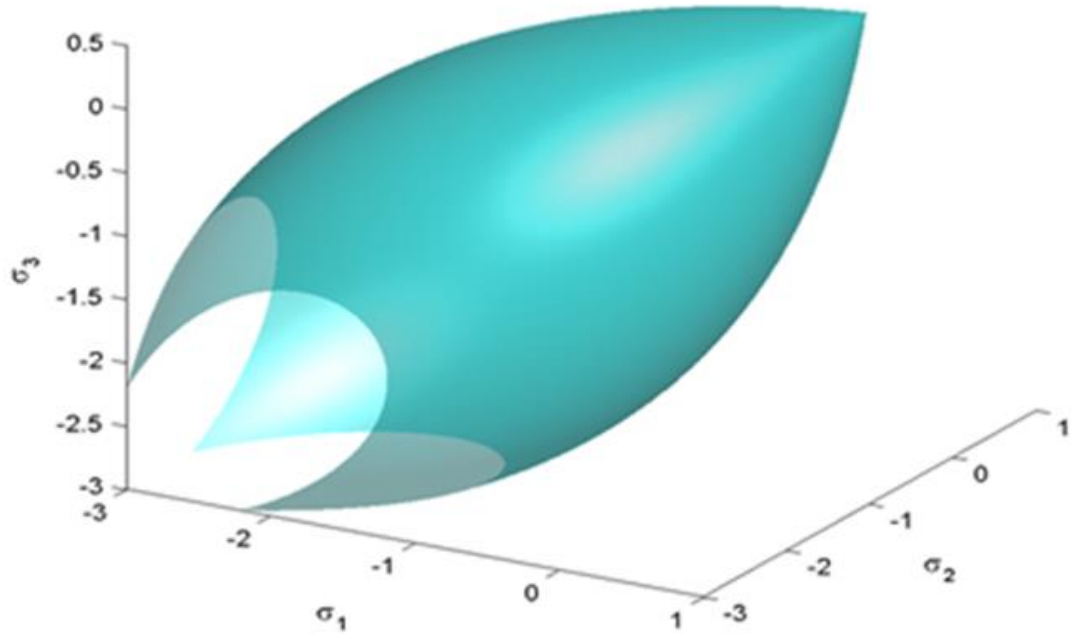

\section{Willam-Warnke Criterion}

Рисунок 1-3D модель поверхности

Критерий Willam-Warnke критерий используется для прогнозирования отказов в бетоне и других когезионно-фрикционных материалов, такие как камень, грунт и керамика. Так же, как критерий Бреслера-Пистера, он зависит только от трех параметров. Он был разработан для описания первоначального разрушения бетона при трехосных испытаниях. Поверхность разрушения является выпуклой, непрерывно 
дифференцируемой и приспособлена для проверки данных в диапазоне низкого сжатия. Материал считаются совершенными упругопластическим (без закалки).

Первоначальный «трехпараметрический» критерий отказа Виллама-Варке был определен как

$$
F_{y}=\sqrt{\frac{3 \tau_{o c t}}{5 f_{c}}+r(\theta)}\left(\left(\frac{1}{f_{t}}-\frac{1}{f_{b}}\right) \sigma_{o c t}-1\right)=0
$$

здесь, $\mathrm{f}_{\mathrm{c}}$ одноосная удельная работа разрыва, $\mathrm{f}_{\mathrm{t}}$ одноосная прочность на растяжение, и $\mathrm{f}_{\mathrm{b}}$ получено от двухосного испытания обжатия. Все параметры положительные. Октаэдрические нормальные и касательные напряжения определяются как обычные.

$$
\begin{gathered}
\sigma_{\text {осt }}=\mathrm{I}_{1} / 3 \text {, and } \tau_{\text {осt }}=\sqrt{2 \mathrm{~J}_{2} / 3} \\
\text { Это можно записать как } \\
\mathrm{F}_{\mathrm{y}}=\sqrt{\mathrm{J}_{2}}+\sqrt{\frac{5}{2}} \mathrm{r}(\theta)\left(\frac{1}{3 \chi} \mathrm{I}_{1}-\mathrm{f}_{\mathrm{c}}\right)=0 \\
\chi=\mathrm{f}_{\mathrm{b}} \mathrm{f}_{\mathrm{t}} /\left(\mathrm{f}_{\mathrm{b}} \mathrm{f}_{\mathrm{c}}-\mathrm{f}_{\mathrm{t}} \mathrm{f}_{\mathrm{c}}\right)
\end{gathered}
$$

Используя угол Лобе $\theta$, безмернаявеличина $\mathrm{r}(\theta)$ определяется как

$$
r(\theta)=\frac{2 r_{c}\left(r_{c}^{2}-r_{t}^{2}\right) \cos \theta+r_{c}\left(2 r_{t}-r_{c}\right) \sqrt{4\left(r_{c}^{2}-r_{t}^{2}\right) \cos ^{2} \theta+5 r_{t}^{2}-4 r_{c} r_{t}}}{4\left(r_{2}^{c}-r_{t}^{2}\right) \cos ^{2} \theta+\left(r_{c}+2 r_{t}\right)^{2}}
$$

Здесь растягивающий и сжимающий меридиан $\mathrm{r}_{\mathrm{t}}$ и $\mathrm{r}_{\mathrm{c}}$ определяются в терминах положительных параметров $\mathrm{f}_{\mathrm{c}}, \mathrm{f}_{\mathrm{b}}$ и $\mathrm{f}_{\mathrm{t}}$ :

$$
\begin{gathered}
r_{t}=\sqrt{\frac{6}{5}} \frac{\mathrm{f}_{b} \mathrm{f}_{t}}{2 \mathrm{f}_{\mathrm{b}} \mathrm{f}_{\mathrm{c}}+\mathrm{f}_{\mathrm{t}} \mathrm{f}_{\mathrm{c}}} \\
\mathrm{r}_{\mathrm{c}}=\sqrt{\frac{6}{5}} \frac{\mathrm{f}_{\mathrm{b}} \mathrm{f}_{\mathrm{t}}}{3 \mathrm{f}_{\mathrm{b}} \mathrm{f}_{\mathrm{t}}+\mathrm{f}_{\mathrm{b}} \mathrm{f}_{\mathrm{c}}-\mathrm{f}_{\mathrm{t}} \mathrm{f}_{\mathrm{c}}}
\end{gathered}
$$

Функции $\mathrm{r}(\theta)$ может быть интерпретирован как угол трения, который зависит от угла Лоде

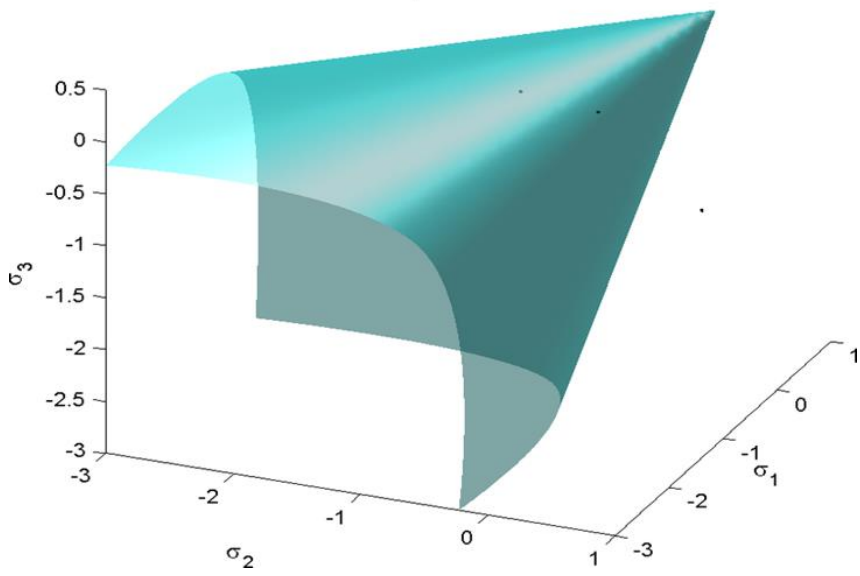

Рисунок 2- 3D модель поверхности 


\section{Ottosen Criterion}

Критерий Оттосена является четырехпараметрическим критерием разрушения, предложенным для кратковременной нагрузки бетона. Она соответствует гладкой выпуклой поверхности разрушения с изогнутыми меридианами, которая открыта в отрицательном (сжимающем) направлении гидростатической оси. Кривая в рі-плоскости изменяется от почти треугольной до более круглой формы с увеличением гидростатического давления. Критерий согласуется с экспериментальными результатами в широком диапазоне напряженных состояний, включая как трехосные испытания вдоль растяжимого и сжимающего меридиана, так и двухосные испытания Критерий Оттосена обычно записывается как

$$
\mathrm{F}_{\mathrm{y}}=\frac{\mathrm{a}}{\mathrm{f}_{\mathrm{c}}} \mathrm{J}_{2}+\lambda(\theta) \sqrt{\mathrm{J}_{2}}+\mathrm{bI}_{1}-\mathrm{f}_{\mathrm{c}}=0
$$

В этой формулировке параметры $\mathrm{a}>0, \mathrm{~b}>0, \mathrm{k} 1>0$ и $\mathrm{k} 2>0$ безразмерны, и $\mathrm{fc}>0$ - одноосная сила сжатия бетона (с положительным знаком).

Функция $\lambda(\theta) \triangleright 0$ (безразмерная) определяется как

$$
\lambda(\theta)=\prec \begin{gathered}
\kappa_{1} \cos \left(\frac{1}{3} \arccos \left(\kappa_{2} \cos (3 \theta)\right)\right) \quad \mathrm{J}_{3}>0 \\
\kappa_{1} \cos \left(\frac{\pi}{3}-\frac{1}{3} \arccos \left(-\kappa_{2} \cos (3 \theta)\right)\right) \quad \mathrm{J}_{3}\langle 0
\end{gathered}
$$

Параметр $\mathrm{k}_{1-}$ фактор размера $\mathrm{k}_{2}$-фактор формы положительна и ограничена $0 \leq \kappa_{2} \leq 1$

Сжимающие и растягивающие сечения (как определено в критерии УилламаВарнке) являются

$$
\begin{gathered}
r_{c}=\frac{1}{\lambda_{3}}=\frac{1}{\lambda\left(\frac{\pi}{3}\right)} \\
r_{t}=\frac{1}{\lambda_{t}} \frac{1}{\lambda(0)}
\end{gathered}
$$

Для бетона соотношение

$$
\lambda_{\mathrm{e}} / \lambda_{\mathrm{e}}=\mathrm{r}_{\mathrm{t}} / \mathrm{r}_{\mathrm{c}}
$$

Обычно лежит между 0.54-0.58

Критерий Ottosen эквивалентно Drucker-Prager, когда $\mathrm{a}=0$ и $\lambda=$ константе. 


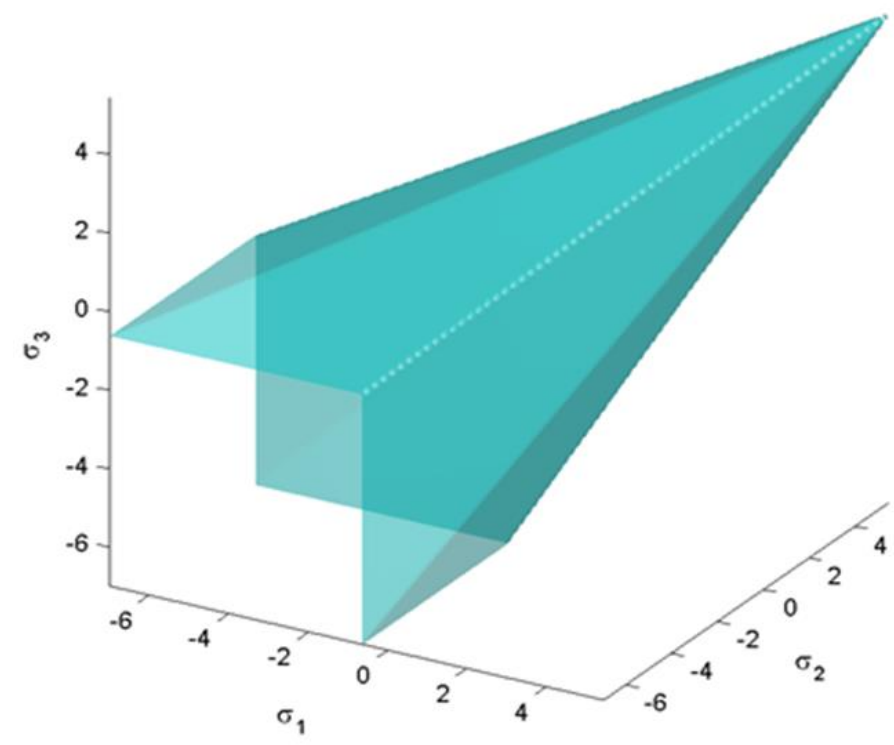

Рисунок 3- 3D модель поверхности

\section{СПИСОК ЛИТЕРАТУРЫ}

1. https://ru.wikipedia.org/wiki/Критерий_прочности_Друкера___Прагера

2. https://en.wikipedia.org/wiki/Willam-Warnke_yield_criterion

3. https://en.wikipedia.org/wiki/Bresler_Pister_yield_criterion

4. Rakenteiden Mekaniikka (Journal of Structural Mechanics) Vol. 47. No 2. 2014. pp. 50 $-66$

5. https://www.comsol.ru/structural-mechanics-module

6. https://www.comsol.ru/geomechanics-module

7. https://books.google.kz/books?id=0DxRDwAAQBAJ\&pg=PA370\&lpg=PA370\&dq=bre sler+pister\&source $=$ bl\&ots $=q 4 b x e V A Y 2 \&$ sig $=$ dqgvyBmJP7 vTpkvrbYU7xj6rLhM\&hl=r $\mathrm{u} \& \mathrm{sa}=\mathrm{X} \& \mathrm{ved}=0 \mathrm{ahUKEwiw1P2du} 4 \mathrm{XaAhVBkCwKHUjxDFw} 4 \mathrm{ChDoAQgvMAI \# v=onep}$ age $\& \mathrm{q}=$ bresler\%20pister $\& \mathrm{f}=$ false

8. http://citeseerx.ist.psu.edu/viewdoc/download?doi=10.1.1.1024.6428\&rep=rep1\&type=p df

9. Журнал инженерная механика (C) обсе / январь 2010 / 5

10. Сопротивление материалов А. В. Александров, В. Д. Потапов, Б. П. Державин ГУП Москва издательство «высшая школа» 2003г.

11. Строительная механика Метод конечных элементов С. И. Трушин 2018 г. 